Supporting Information

\title{
Sub-100 fs Charge Separation and Subsequent Diffusive Solvation Observed for Asymmetric Bianthryl Derivative in Ionic Liquid
}

Eisuke Takeuchi, Masayasu Muramatsu, Tetsuro Katayama, Yusuke Yoneda, Syoji Ito, Yutaka Nagasawa*, and Hiroshi Miyasaka*

Table of Contents

Figure S1. TA spectra of CBA in $n$-hexane.

Figure S2. Decay associated spectra (DAS) in polar solvents. page

S2

S3 

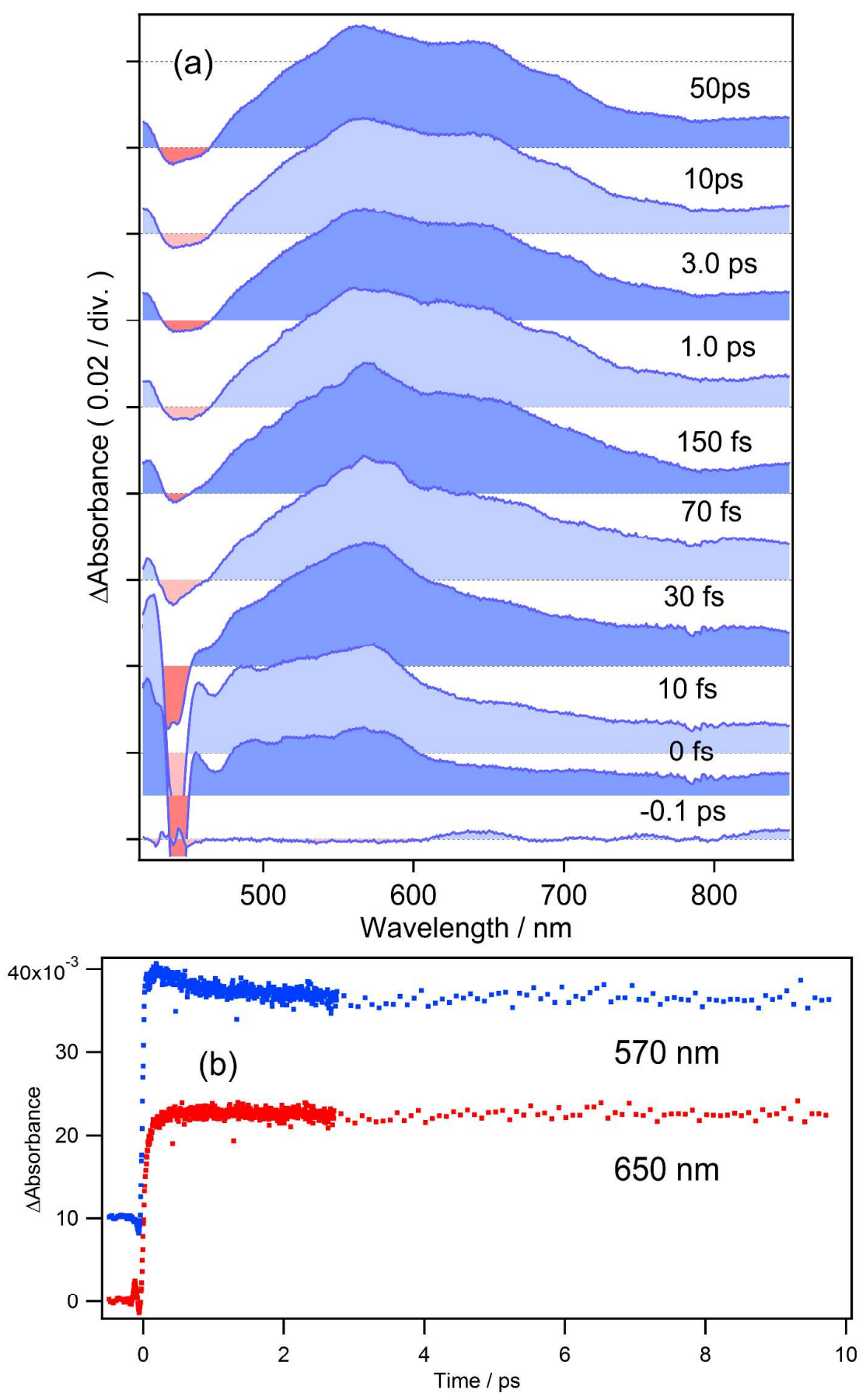

Figure S1. (a) Transient absorption (TA) spectra of 10-cyano-9,9'-bianthryl (CBA) in $n$-hexane excited at $420 \mathrm{~nm}$ with pulse duration of $\sim 30 \mathrm{fs}$. (b) Time dependence of the differential absorption at $570 \mathrm{~nm}$ and $650 \mathrm{~nm}$. The time constant for the decay seen at $570 \mathrm{~nm}$ is $820 \pm 70 \mathrm{fs}$. 

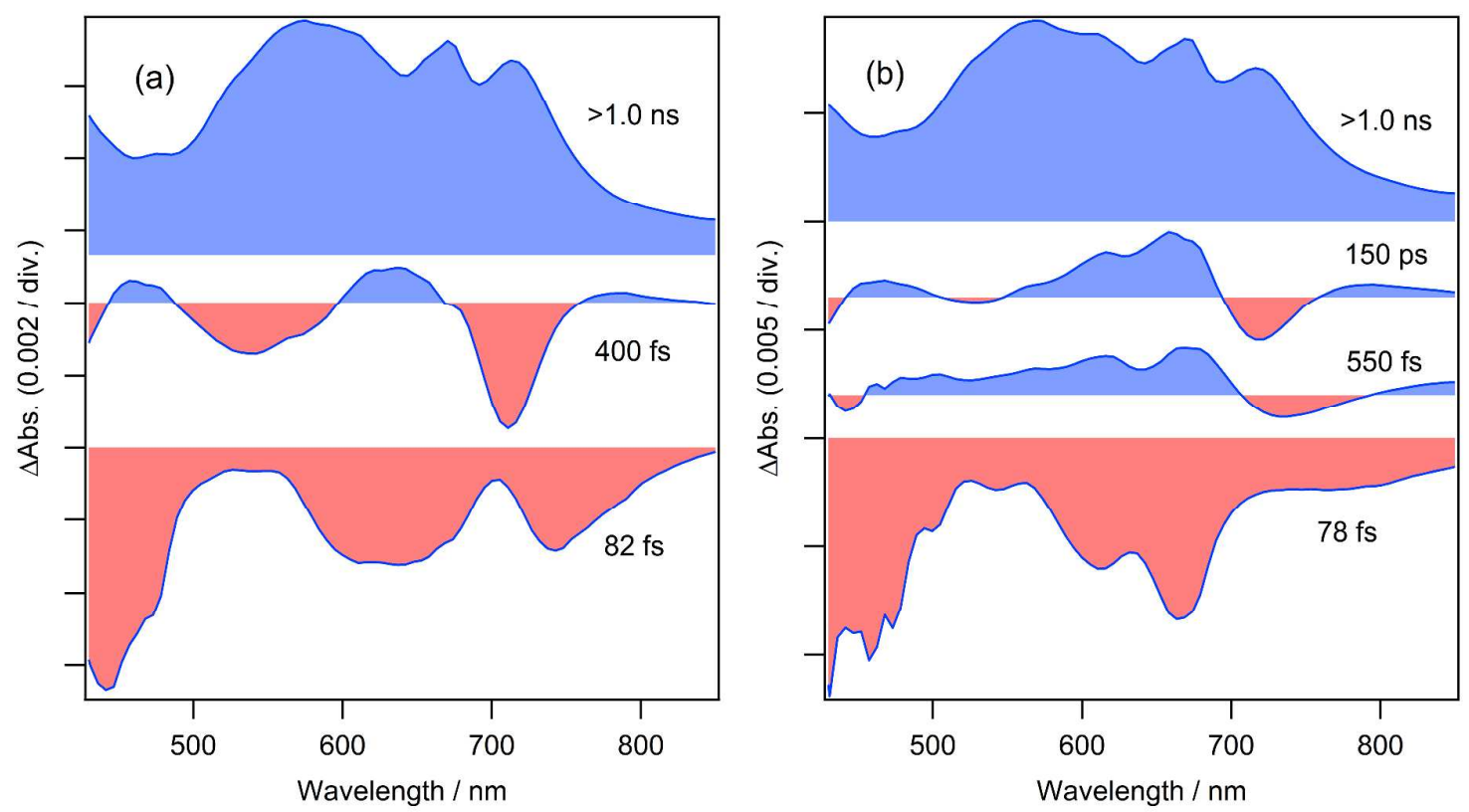

Figure S2. Decay associated spectra (DAS) obtained by the global fitting of the transient absorption (TA) spectra of 10-cyano-9,9'-bianthryl (CBA) in (a) acetonitrile (Acn) and in (b) DemeBF$_{4}$.

Global analysis was performed from delay of $30 \mathrm{fs}$ to avoid the effect of coherent spike at the time origin and the obtained decay associated spectra (DAS) are presented in Figure S1. For the Acn solution, three components with time constants of $82 \mathrm{fs}, 400 \mathrm{fs}$ and $>1.0$ ns were obtained (Figure S1a), while in the DemeBF 4 solution, four components with time constants of $78 \mathrm{fs}, 550 \mathrm{fs}, 150 \mathrm{ps}$, and >1.0 ns were obtained (Figure S1b). In the DemeBF 4 solution, the DAS with time constant of $78 \mathrm{fs}$ has a strong negative peak at $660 \mathrm{~nm}$ corresponding to the rise of the CS state seen in Figure 2 with only a weak featureless negative band in the range of $>700 \mathrm{~nm}$. Meanwhile for the Acn solution, featureless broad negative band appears at 590-680 nm with a significant negative peak at $740 \mathrm{~nm}$ (Figure S1a). These spectral discrepancies indicate that, in Acn, the primary CS process is somewhat mixed with the secondary structural relaxation process because the time scales are not well separated in Acn compared to those in the ionic liquid solution. The strong negative band in the range of $<500$ $\mathrm{nm}$ represents the ultrafast quenching of the stimulated emission. The time constants of $78 \mathrm{fs}$ and $82 \mathrm{fs}$ are somewhat longer than that obtained by the single wavelength probing at $670 \mathrm{~nm}$ (Figure 4a), because they are averaged out for the entire wavelength range of 430-850 nm by the global analysis. The secondary structural relaxation process is characterized by the rise of the peak at $710 \mathrm{~nm}$ in Acn with a time constant of $700 \mathrm{fs}$, while it is separated into two processes in DemeBF 4 with time constants of 550 fs and 150 ps reflecting the 
multiexponential nature of the dynamics in the viscous ionic liquid. Note that the minimum of the negative band in the range of $>700 \mathrm{~nm}$ shifts from $740 \mathrm{~nm}$ to $710 \mathrm{~nm}$ in Acn and that in DemeBF$_{4}$ shifts from $760 \mathrm{~nm}$ to $740 \mathrm{~nm}$ and $720 \mathrm{~nm}$ with time, indicating that this is not a simple species-to-species reaction but an energy relaxation process. 